TRANSACTIONS OF THE

AMERICAN MATHEMATICAL SOCIETY

Volume 352 Number 7 , Pages 3265-328

S 0002-9947(99)02573-8

Article electronically published on November 18, 1999

\title{
SHARP WEIGHTED INEQUALITIES FOR THE VECTOR-VALUED MAXIMAL FUNCTION
}

\author{
CARLOS PÉREZ
}

Abstract. We prove in this paper some sharp weighted inequalities for the vector-valued maximal function $\bar{M}_{q}$ of Fefferman and Stein defined by

$$
\bar{M}_{q} f(x)=\left(\sum_{i=1}^{\infty}\left(M f_{i}(x)\right)^{q}\right)^{1 / q},
$$

where $M$ is the Hardy-Littlewood maximal function. As a consequence we derive the main result establishing that in the range $1<q<p<\infty$ there exists a constant $C$ such that

$$
\int_{\mathbf{R}^{n}} \bar{M}_{q} f(x)^{p} w(x) d x \leq C \int_{\mathbf{R}^{n}}|f(x)|_{q}^{p} M^{\left[\frac{p}{q}\right]+1} w(x) d x .
$$

Furthermore the result is sharp since $M^{\left[\frac{p}{q}\right]+1}$ cannot be replaced by $M^{\left[\frac{p}{q}\right]}$. We also show the following endpoint estimate

$$
w\left(\left\{x \in \mathbf{R}^{n}: \bar{M}_{q} f(x)>\lambda\right\}\right) \leq \frac{C}{\lambda} \int_{\mathbf{R}^{n}}|f(x)|_{q} M w(x) d x,
$$

where $C$ is a constant independent of $\lambda$.

\section{Motivation AND DESCRIPTION OF THE MAIN RESUlts}

The purpose of this paper is to obtain some sharp weighted inequalities for the vector-valued maximal function $\bar{M}_{q}$ which are not within the scope of the standard $A_{p}$ theory for vector-valued singular integrals as can be found in [RRT]. We start with a review of some of the classical estimates and then we shall state the main results.

1.1. Background. Let $M$ be the Hardy-Littlewood maximal function and let $\bar{M}_{q}$ be the vector-valued maximal operator defined by

$$
\bar{M}_{q} f(x)=\left(\sum_{i=1}^{\infty}\left(M f_{i}(x)\right)^{q}\right)^{1 / q} .
$$

This nonlinear operator was introduced by C. Fefferman and E. M. Stein in [FS] as a generalization of both the (scalar) maximal function $M$ and the classical integral of Marcinkiewicz and since then it has played an important role in the development of modern Harmonic Analysis.

We recall the two basic estimates obtained in [FS] for $1<q<\infty$ :

Received by the editors May 19, 1997.

1991 Mathematics Subject Classification. Primary 42B20, 42B25, 42B15.

This work was partially supported by DGICYT grant PB940192, Spain. 
- Let $1<p<\infty$, then there exists a constant $C$ such that

$$
\int_{\mathbf{R}^{n}} \bar{M}_{q} f(x)^{p} d x \leq C \int_{\mathbf{R}^{n}}|f(x)|_{q}^{p} d x .
$$

- The following weak type $(1,1)$ estimate holds: there exists a constant $c$ such that

$$
\sup _{\lambda>0} \lambda\left|\left\{x \in \mathbf{R}^{n}: \bar{M}_{q} f(x)>\lambda\right\}\right| \leq C \int_{\mathbf{R}^{n}}|f(x)|_{q} d x .
$$

We are using here the notation $|f(x)|_{q}=\left(\sum_{i=1}^{\infty}\left|f_{i}(x)\right|^{q}\right)^{1 / q}=\|f(x)\|_{\ell^{q}}$.

Another fundamental generalization of the maximal theorem is due to B. Muckenhoupt [M] who gave a characterization of the following "weighted norm inequality"

$$
\int_{\mathbf{R}^{n}} M f(x)^{p} w(x) d x \leq c \int_{\mathbf{R}^{n}}|f(x)|^{p} w(x) d x,
$$

in terms of the $A_{p}$ condition of Muckenhoupt: there exists a positive constant $c$ such that for all cubes $Q$

$$
A_{p} \quad \frac{1}{|Q|} \int_{Q} w(y) d y\left(\frac{1}{|Q|} \int_{Q} w(y)^{1-p^{\prime}} d y\right)^{p-1} \leq c .
$$

It is also well known that the $A_{p}$ condition (4) also characterizes all the weights $w$ for which the weighted vector-valued inequality holds

$$
\int_{\mathbf{R}^{n}} \bar{M}_{q} f(x)^{p} w(x) d x \leq C \int_{\mathbf{R}^{n}}|f(x)|_{q}^{p} w(x) d x .
$$

This result is due to K. F. Andersen and R. T. John AJ], and to V. Kokilashvili [K]. There are by now three ways of proving (5):

- Refining the argument of Fefferman and E. M. Stein in [FS] as done in [AJ] and $[\mathrm{K}]$.

- Looking at $\bar{M}_{q}$ as a vector-valued singular integral with operator-valued kernel satisfying a pointwise gradient condition as can be found in [RRT].

- By applying the extrapolation theory of J. Garcia-Cuerva and J. L. Rubio de Francia as mentioned in GCRdF p. 521 which yields a simple proof.

In this paper we investigate the two weight problem for the vector-valued maximal function $\bar{M}_{q}$

$$
\int_{\mathbf{R}^{n}} \bar{M}_{q} f(x)^{p} w(x) d x \leq C \int_{\mathbf{R}^{n}}|f(x)|_{q}^{p} v(x) d x,
$$

for which none of the above approaches works.

Recall that there is a characterization due to E. Sawyer $[\mathbf{S}]$ of the two weight problem in the scalar situation, the following weighted inequality

$$
\int_{\mathbf{R}^{n}} M f(x)^{p} w(x) d x \leq C \int_{\mathbf{R}^{n}}|f(x)|^{p} v(x) d x
$$

holds if and only if there exists a constant $c$ such that for all cubes $Q$

$$
S_{p} \quad \int_{Q} M\left(v^{1-p^{\prime}} \chi_{Q}\right)(y)^{p} w(y) d y \leq c \int_{Q} v(y)^{1-p^{\prime}} d y .
$$

The range $p \leq q$ for (6) is easy to handle since it coincides with the scalar situation. Indeed, if $p \leq q$ we claim that the $S_{p}$ condition is necessary and sufficient 
for (6). It is clear that condition (8) is necessary. If we assume (8) we see that (6) is immediate for both $q=p$ and $q=\infty$. Then the case $1<p<q<\infty$ follows by interpolation for "linearizable operators" in the vector-valued context (cf. the argument given in [GCRdF] p. 482).

Although we shall give a full characterization of (6) in Theorem 2.3 we are more interested in estimates of the form

$$
\int_{\mathbf{R}^{n}} \bar{M}_{q} f(x)^{p} w(x) d x \leq C \int_{\mathbf{R}^{n}}|f(x)|_{q}^{p} N w(x) d x,
$$

where $N$ is an appropriate (scalar) maximal type operator. Needless to say that the prototypical estimate that we have in mind is the Fefferman-Stein weighted inequality

$$
\int_{\mathbf{R}^{n}} M f(x)^{p} w(x) d x \leq C \int_{\mathbf{R}^{n}}|f(x)|^{p} M w(x) d x
$$

which yields, as it is well known, the unweighted vector-valued estimate (1) when $p>q$.

One of the main observations that follows from our results is that $\bar{M}_{q}$ does not verify a similar inequality to 10 on the range $p>q$ (cf. the first remark after Theorem 1.1).

Inequalities of the type (10) reflect how singular the operator under study is. This can be seen for instance with the following sharp inequalities for singular integrals obtained in $[\mathrm{P} 2$ for $p>1$ generalizing some previous estimates obtained by $\mathrm{M}$. Wilson in the range $1<p \leq 2$ Wil2]:

Let $T$ be any Calderón-Zygmund operator, and let $1<p<\infty$. Then there exists a constant $C$ such that

$$
\int_{\mathbf{R}^{n}}|T f(x)|^{p} w(x) d x \leq C \int_{\mathbf{R}^{n}}|f(x)|^{p} M^{[p]+1} w(x) d x,
$$

with $C$ independent of $w$ and $f$. Furthermore, the estimate is sharp since it does not hold for $M^{[p]}$. Here $M^{k}=M \circ \stackrel{(k)}{.} \circ M, k=1,2, \cdots$, denotes the Hardy-Littlewood maximal operator $M$ iterated $k$ times.

Another example which stresses our point of view is related to the classical Area function. This nonlinear operator is defined by the integral

$$
S_{\varphi}(f)(x)=\left(\int_{B_{t}(x)}\left|f * \varphi_{t}(y)\right|^{2} \frac{d t d y}{t^{n+1}}\right)^{1 / 2},
$$

where $\varphi \in C_{0}^{\infty}$ with $\int \varphi=0$ and $\varphi_{t}(x)=t^{-n} \varphi\left(\frac{x}{t}\right), t>0$. Then the Area function satisfies the following inequality:

Let $1<p \leq 2$, then there exists a constant $C$ such that

$$
\int_{\mathbf{R}^{n}} S_{\varphi}(f)(x)^{p} w(x) d x \leq C \int_{\mathbf{R}^{n}}|f(x)|^{p} M w(x) d x .
$$

Furthermore, this inequality is false for $p>2$.

The case $p=2$ was first obtained by A. Chang, M. Wilson, and T. Wolff in CWW] and for $1<p<2$ by S. Chanillo and R. Wheeden in [CW] as well as the counterexample for $p>2$. See also the work by M. Wilson Wil1- Wil4. 
1.2. Main results. Motivated by the theorems mentioned above we state now the main result of the paper.

Theorem 1.1. Let $1<q<p<\infty$.

a) There exists a constant $C$ such that

$$
\int_{\mathbf{R}^{n}} \bar{M}_{q} f(x)^{p} w(x) d x \leq C \int_{\mathbf{R}^{n}}|f(x)|_{q}^{p} M^{\left[\frac{p}{q}\right]+1} w(x) d x,
$$

for all locally integrable functions $f, w \geq 0$.

b) Part a) is sharp since there exists no constant $C$ such that

$$
\int_{\mathbf{R}^{n}} \bar{M}_{q} f(x)^{p} w(x) d x \leq C \int_{\mathbf{R}^{n}}|f(x)|_{q}^{p} M^{\left[\frac{p}{q}\right]} w(x) d x,
$$

for all locally integrable functions $f, w \geq 0$. Likewise, the corresponding weak type $(p, p)$ estimate is false.

We now make the following remarks.

(a) It follows from part b) of Theorem 1.1 that the vector-valued analogue of the Fefferman-Stein inequality (10)

$$
\int_{\mathbf{R}^{n}} \bar{M}_{q} f(x)^{p} w(x) d x \leq C \int_{\mathbf{R}^{n}}|f(x)|_{q}^{p} M w(x) d x
$$

is false in general in the range $p>q$.

(b) If we look at the proof of (13), we see that we can refine such an inequality by replacing $M^{\left[\frac{p}{q}\right]+1}$ by $M_{L(\log L)^{\frac{p}{q}-1+\epsilon}}, \epsilon>0$, or by $M_{A}$ where $A$ satisfies

$$
\int_{c}^{\infty}\left(\frac{t}{A(t)}\right)^{\left(\frac{p}{q}\right)^{\prime}-1} \frac{d t}{t}<\infty
$$

See Section 3 for the appropriate definition of the maximal type function $M_{A}$.

(c) We emphasize on the fact that there is no assumption on $w$ other than local integrabilty. In fact, if we assume that $w \in A_{\infty}$, then (15) holds being false in general. Indeed, by the Lebesgue differentation theorem we have

$$
\begin{aligned}
\int_{\mathbf{R}^{n}} \bar{M}_{q} f(x)^{p} w(x) d x & \leq \int_{\mathbf{R}^{n}} \bar{M}_{q} f(x)^{p} M w(x) d x \\
& \leq C \int_{\mathbf{R}^{n}}|f(x)|_{q}^{p} M w(x) d x,
\end{aligned}
$$

where in the last inequality we have used the $A_{p}$ result for $\bar{M}_{q}$ (5) since $M w \in A_{1}$ by standard results (see the last part of Section 4 ).

Also, we may replace $M^{\left[\frac{p}{q}\right]+1} w$ by the $A_{1}$ weight $M\left(w^{r}\right)(x)^{1 / r}, r>1$, by applying again (5). However, the later class of weights (essentially the class $A_{1}$ ) are pointwise larger than the non- $A_{\infty}$ weights $M^{k} w$ since it may be shown using standard theory that:

For each integer $k=1,2, \cdots$, each $r>1$ and each locally integrable function $f$, we have the following pointwise inequality for all $x \in \mathbf{R}^{n}$ :

$$
w(x) \leq M^{k} w(x) \leq\left[M\left(w^{r}\right)^{1 / r}\right]_{A_{1}}^{k-1} M\left(w^{r}\right)(x)^{1 / r} .
$$

Here $[w]_{A_{1}}$ denotes the "norm" of $w \in A_{1}$, namely the smallest constant $C$ such that $M w \leq C w$. 
(d) Theorem 1.1 indicates that $\bar{M}_{q}$ behaves more as a singular integral operator rather than as a maximal operator. However, we want to emphasize the fact that inequality (13) does not fit within the scope of the theory of vector-valued singular integrals as developed by J. L. Rubio de Francia, F. J. Ruiz and J. L. Torrea in [RRT] where the pioneering work [BCP] was updated. In [RRT], the operator $\bar{M}_{q}$, as well as many other nonlinear operators such as the Area function $S_{\varphi}$, are seen as singular integrals taking values in an appropriate Banach space. Using this point of view, it is possible to translate to this more general context the one weight scalar $A_{p}$ theory at least for any vector-valued singular integral with sufficiently smooth kernel. However, this is not the case of (13) (nor of [12) since the result for the (scalar) Hilbert transform (11) is worse than (13) indicating that the operator $\bar{M}_{q}$ is less singular than $H$.

The proof of the positive part of Theorem 1.1 does not follow the scheme used in $\mathrm{P} 2$ to treat singular integrals since we cannot dualize (13). We shall derive (13) as a consequence of a characterization for the two weight problem given in Theorem 2.3. The condition we obtain is a blend of Sawyer's condition $S_{p}$ together with Rubio de Francia's characterization of vector-valued inequalities for sublinear operators as can be found in GCRdF, Chapter VI.

It should be mentioned that Y. Rakotondratsimba has obtained in $[\mathrm{R}]$ a different characterization of the two weight problem which is much closer in spirit to Sawyer's condition $S_{p}$.

1.3. Sharp sufficient conditions close to $A_{p}$. In this section we take up the two weight problem for $\bar{M}_{q}$ that we write in the following more convenient form

$$
\int_{\mathbf{R}^{n}}\left(w(x) \bar{M}_{q} f(x)\right)^{p} d x \leq C \int_{\mathbf{R}^{n}}\left(v(x)|f(x)|_{q}\right)^{p} d x .
$$

The task is to provide sharp sufficient conditions on the weights "close" in structure to the $A_{p}$ condition.

Let us briefly review some results related to the scalar situation

$$
\int_{\mathbf{R}^{n}}(w(x) M f(x))^{p} d x \leq c \int_{\mathbf{R}^{n}}(v(x)|f(x)|)^{p} d x .
$$

It is well known that the necessary $A_{p}$ condition for this problem

$$
\left(\frac{1}{|Q|} \int_{Q} w(x)^{p} d x\right)^{1 / p}\left(\frac{1}{|Q|} \int_{Q} v(x)^{-p^{\prime}} d x\right)^{1 / p^{\prime}} \leq c
$$

is not sufficient, and that the correct necessary and sufficient condition is, as we mentioned above, Sawyer's condition which with our normalization on the weights has the following form:

$$
\int_{Q}\left(w(y) M\left(v^{-p^{\prime}} \chi_{Q}\right)(y)\right)^{p} d y \leq C \int_{Q} v(y)^{-p^{\prime}} d y
$$

The drawback of this condition is that it involves the operator $M$ itself, and it would be interesting to obtain sufficient conditions close in form to the $A_{p}$ condition (19). Perhaps, the first result in that direction was obtained by C. Neugebauer in [N]. He noticed that if $(w, v)$ is a couple of weights such that for some $r>1$

$$
\left(\frac{1}{|Q|} \int_{Q} w(y)^{p r} d y\right)^{1 / p r}\left(\frac{1}{|Q|} \int_{Q} v(y)^{-p^{\prime} r} d y\right)^{1 / p^{\prime} r} \leq c
$$


for all cubes $Q$, then

$$
\int_{\mathbf{R}^{n}}(w(y) M f(y))^{p} d y \leq c \int_{\mathbf{R}^{n}}(v(y)|f(y)|)^{p} d y .
$$

In fact Neugebauer proves that (20) is equivalent to showing that there is an $A_{p}$ weight inserted (pointwise) between $w^{p}$ and $v^{p}$ and the result follows trivially. This problem has been considered in [P1] where it is shown that such a strong condition is not needed. In particular it is not necessary to "bump" the left weight $w$ and that much less than a power "bump" is required on the right weight $v$ to get the result. We extract the following result from [P1]. Recall that for a given Young function $A$ and a cube $Q$ on $\mathbf{R}^{n}$ we defined the $A$-average of a function $f$ over $Q$ by

$$
\|f\|_{A, Q}=\inf \left\{\lambda>0: \frac{1}{|Q|} \int_{Q} A\left(\frac{|f(y)|}{\lambda}\right) d y \leq 1\right\} .
$$

Theorem $1.2([\mathrm{P} 1])$. Let $1<p<\infty$, and let $B$ be a doubling Young function such that

$$
\int_{c}^{\infty}\left(\frac{t^{p^{\prime}}}{B(t)}\right)^{p-1} \frac{d t}{t}<\infty
$$

for some positive constant $c$. Let $(w, v)$ be a couple of weights such that there is a positive constant $K$ for which

$$
\left(\frac{1}{|Q|} \int_{Q} w(y)^{p} d y\right)^{1 / p}\left\|v^{-1}\right\|_{B, Q} \leq K
$$

for all cubes $Q$. Then

$$
\int_{\mathbf{R}^{n}}(w(y) M f(y))^{p} d y \leq c \int_{\mathbf{R}^{n}}(v(y) f(y))^{p} d y
$$

for all nonnegative functions $f$.

As we may expect we need to consider stronger conditions on the weights to get corresponding results for $\bar{M}_{q}$ in the range $p>q$. In particular we need to "bump" the left weight $w$ as well since otherwise the result is false as the counterexample $(w, M w)$ in (15) shows. Indeed, observe that this pair of weights satisfies (23) for any cube $Q$ and any Young function $B$ :

$$
\begin{gathered}
\left(\frac{1}{|Q|} \int_{Q} w\right)^{1 / p}\left\|(M w)^{-1 / p}\right\|_{B, Q} \leq\left(\frac{1}{|Q|} \int_{Q} w\right)^{1 / p}\left\|\left(\frac{1}{|Q|} \int_{Q} w\right)^{-1 / p}\right\|_{B, Q} \\
=\left(\frac{1}{|Q|} \int_{Q} w\right)^{1 / p}\left(\frac{1}{|Q|} \int_{Q} w\right)^{-1 / p}\|1\|_{B, Q}=1
\end{gathered}
$$

since $\frac{1}{|Q|} \int_{Q} w \leq M w(x)$ for $x \in Q$.

Theorem 1.3. Let $1<q<p<\infty$, and let $r=\frac{p}{q}$. Let $A, B$ be doubling Young functions such that both

$$
\int_{c}^{\infty}\left(\frac{t^{r}}{A(t)}\right)^{r^{\prime}-1} \frac{d t}{t} \quad \text { and } \quad \int_{c}^{\infty}\left(\frac{t^{q^{\prime}}}{B(t)}\right)^{q-1} \frac{d t}{t},
$$


are finite for some positive constant $c$, that is, $\bar{A} \in B_{r^{\prime}}$ and $\bar{B} \in B_{q}$ (see Definition 3.1). Let $(w, v)$ be a couple of weights such that there is a positive constant $K$ for which

$$
\left\|w^{q}\right\|_{A, Q}^{1 / q}\left\|v^{-1}\right\|_{B, Q} \leq K,
$$

for all cubes $Q$. Then the two weighted vector-valued inequality

$$
\left\|\left(\sum_{i=0}^{\infty}\left(w M f_{i}\right)^{q}\right)^{1 / q}\right\|_{L^{p}\left(\mathbf{R}^{n}\right)} \leq C\left\|\left(\sum_{i=0}^{\infty}\left|v f_{i}\right|^{q}\right)^{1 / q}\right\|_{L^{p}\left(\mathbf{R}^{n}\right)}
$$

holds for all $f_{i}$.

Some interesting examples are given by $A(t) \approx t^{r}(\log t)^{r-1+\delta}$ and $B(t) \approx$ $t^{q^{\prime}}(\log t)^{q^{\prime}-1+\delta}$ with $\delta>0$.

1.4. Endpoint estimates. Although the operator $\bar{M}_{q}$ is, to some extent, more closely related to a singular integral this is not the case when we look at endpoint estimates such as the following.

Theorem 1.4. There exists a constant $C$ such that for each weight $w$ and for all $\lambda>0$

$$
w\left(\left\{x \in \mathbf{R}^{n}: \bar{M}_{q} f(x)>\lambda\right\}\right) \leq \frac{C}{\lambda} \int_{\mathbf{R}^{n}}|f(x)|_{q} M w(x) d x .
$$

This result reflects once again that sharp results for $\bar{M}_{q}$ are independent from the theory of vector-valued singular integrals since we do not know whether the (scalar) Hilbert transform $H$ satisfies

$$
w\left(\left\{x \in \mathbf{R}^{n}:|H f(x)|>\lambda\right\}\right) \leq \frac{C}{\lambda} \int_{\mathbf{R}^{n}}|f(x)| M w(x) d x .
$$

See [P2] for sharp results.

There exists an interesting relationship between (28) and a possible vector-valued version of the classical Besicovitch lemma. We shall formulate this as a conjecture. $M_{w}^{c}$ denotes the weighted centered maximal function.

Conjecture 1.5.

$$
w\left(\left\{x \in \mathbf{R}^{n}:\left(\sum_{i=1}^{\infty}\left(M_{w}^{c} f_{i}(x)\right)^{q}\right)^{1 / q}>\lambda\right\}\right) \leq \frac{C}{\lambda} \int_{\mathbf{R}^{n}}|f(x)|_{q} w(x) d x .
$$

One can show (cf. Section 6) that if the conjecture were true, then the inequality (28) follows immediately.

\section{ACKNOWLEDGEMENTS}

The author is very grateful to A. Vargas for several conversations concerning the problems considered in this paper. 


\section{A Characterization of the two Weight PROBlem}

The purpose of this section is to give a characterization of the two weight problem for the vector-valued maximal function $\bar{M}_{q}$. We recall that the case $1<p \leq q$ is characterized by means of Sawyer's condition $S_{p}$. The main result is Theorem 2.3 For the proof of this theorem it will be more efficient to work within a more general context. Let $\mathcal{B}$ be a basis in $\mathbf{R}^{n}$, and by this we mean a collection of open sets in $\mathbf{R}^{n}$. We say that $w$ is a weight associated to the basis $\mathcal{B}$ if $w$ is a nonnegative measurable function in $\mathbf{R}^{n}$ such that $w(B)=\int_{B} w(y) d y<\infty$ for each $B$ in $\mathcal{B}$. $M_{\mathcal{B}, w}$ is the corresponding maximal operator defined by

$$
M_{\mathcal{B}, w} f(x)=\sup _{x \in B} \frac{1}{w(B)} \int_{B}|f(y)| w(y) d y
$$

if $x \in \bigcup_{B \in \mathcal{B}}$ and $M_{\mathcal{B}, w} f(x)=0$ otherwise. If $w \equiv 1$, we just write $M_{\mathcal{B}} f(x)$.

Proposition 2.1. Let $1<q<p<\infty$, and let $r=\frac{p}{q}$. Suppose that $M_{\mathcal{B}, \sigma}: L_{\ell^{q}}^{p}(\sigma) \rightarrow L_{\ell^{q}}^{p}(\sigma)$ where $\sigma=v^{1-p^{\prime}}$.

Then the two weight vector-valued inequality

$$
\left\|\left(\sum_{i=0}^{\infty}\left(M_{\mathcal{B}} f_{i}\right)^{q}\right)^{1 / q}\right\|_{L^{p}(w)} \leq C\left\|\left(\sum_{i=0}^{\infty}\left|f_{i}\right|^{q}\right)^{1 / q}\right\|_{L^{p}(v)}
$$

holds if and only if there exists a constant $c$ such that for each $g \in L^{r^{\prime}}\left(\mathbf{R}^{n}\right)$ we can find $G \in L^{r^{\prime}}\left(\mathbf{R}^{n}\right)$ with $\|G\|_{L^{r^{\prime}}\left(\mathbf{R}^{n}\right)} \leq\|g\|_{L^{r^{\prime}}\left(\mathbf{R}^{n}\right)}$ such that

$$
\int_{\Omega} M_{\mathcal{B}}\left(\sigma \chi_{\Omega}\right)(x)^{q} w(x)^{1 / r} g(x) d x \leq c \int_{\Omega} \sigma(x)^{1 / r} G(x) d x,
$$

for every set $\Omega$ which is a union of sets in $\mathcal{B}$.

Proof. We first show that condition (32) is necessary. First observe that inequality (31) is equivalent to

$$
\left\|\left(\sum_{i=0}^{\infty}\left(w^{1 / p} M_{\mathcal{B}}\left(\frac{f_{i}}{v^{1 / p}}\right)\right)^{q}\right)^{1 / q}\right\|_{L^{p}\left(\mathbf{R}^{n}\right)} \leq c\left\|\left(\sum_{i=0}^{\infty}\left|f_{i}\right|^{q}\right)^{1 / q}\right\|_{L^{p}\left(\mathbf{R}^{n}\right)} .
$$

Now, by Rubio de Francia's theorem (cf. GCRdF], p. 555) this estimate is equivalent to showing that for each $g \in L^{r^{\prime}}\left(\mathbf{R}^{n}\right)$ there exists $G \in L^{r^{\prime}}\left(\mathbf{R}^{n}\right)$ with $\|G\|_{L^{r^{\prime}}\left(\mathbf{R}^{n}\right)}$ $\leq\|g\|_{L^{r^{\prime}}\left(\mathbf{R}^{n}\right)}$ and

$$
\int_{\mathbf{R}^{n}}\left(w(y)^{1 / p} M_{\mathcal{B}}\left(\frac{f}{v^{1 / p}}\right)(y)\right)^{q} g(y) d y \leq C \int_{\mathbf{R}^{n}}|f(y)|^{q} G(y) d y,
$$

for all $f$, or what is the same

$$
\int_{\mathbf{R}^{n}} M_{\mathcal{B}}(f)(y)^{q} w(y)^{1 / r} g(y) d y \leq C \int_{\mathbf{R}^{n}}|f(y)|^{q} v(y)^{1 / r} G(y) d y,
$$

for all $f$. Testing this inequality with $f=\sigma \chi_{\Omega}=v^{1-p^{\prime}} \chi_{\Omega}$ gives the necessary condition (32). 
To prove the sufficiency of (32) we use that $L^{r}$ and $L^{r^{\prime}}$ are dual spaces. We adapt the basic ideas from GCRdF. If we define $I$ as

$$
I=\left\|\left(\sum_{i=0}^{\infty}\left(w^{1 / p} M_{\mathcal{B}} f_{i}\right)^{q}\right)^{1 / q}\right\|_{L^{p}\left(\mathbf{R}^{n}\right)}^{q},
$$

then

$$
I=\sum_{i=0}^{\infty} \int_{\mathbf{R}^{n}} M_{\mathcal{B}}\left(f_{i}\right)(y)^{q} w(y)^{1 / r} g(y) d y
$$

for some $g \in L^{r^{\prime}}\left(\mathbf{R}^{n}\right)$ with unit norm. Fix $i$, and for each integer $k$ consider the set $E_{k}^{i}=\left\{y \in \mathbf{R}^{n}: 2^{k}<M_{\mathcal{B}} f_{i}(y) \leq 2^{k+1}\right\}$. From the definition of $M_{\mathcal{B}}, E_{k}^{i} \subset \bigcup_{j} B_{k, j}^{i}$, where $B_{k, j}^{i} \in \mathcal{B}$ satisfies

$$
2^{k}<\frac{1}{\left|B_{k, j}^{i}\right|} \int_{B_{k, j}^{i}} f_{i}(y) d y .
$$

Define now $E_{k, 1}^{i}=B_{k, 1}^{i} \cap E_{k}^{i}$, and for $j>1 E_{k, j}^{i}=\left(B_{k, j}^{i} \backslash \bigcup_{s<j} B_{k, s}^{i}\right) \cap E_{k}^{i}$. For any fixed $k$, each of the sets $E_{k}^{i}$ is the disjoint union of the sets $E_{k, j}^{i}$. We now can write

$$
\begin{gathered}
\int_{\mathbf{R}^{n}} M_{\mathcal{B}} f_{i}(y)^{q} w(y)^{1 / r} g(y) d y=\sum_{k} \int_{E_{k}^{i}} M_{\mathcal{B}} f_{i}(y)^{q} w(y)^{1 / r} g(y) d y \\
=\sum_{k, j} \int_{E_{k, j}^{i}} M_{\mathcal{B}} f_{i}(y)^{q} w(y)^{1 / r} g(y) d y \leq 2^{q} \sum_{k, j} 2^{k q}\left(w^{1 / r} g\right)\left(E_{k, j}^{i}\right) \\
\leq C \sum_{k, j}\left(w^{1 / r} g\right)\left(E_{k, j}^{i}\right)\left(\frac{1}{\left|B_{k, j}^{i}\right|} \int_{B_{k, j}^{i}} f_{i}(y) d y\right)^{q} \\
=c \sum_{k, j}\left(w^{1 / r} g\right)\left(E_{k, j}^{i}\right)\left(\frac{1}{\left|B_{k, j}^{i}\right|} \int_{B_{k, j}^{i}} \sigma(y) d y\right)^{q}\left[\frac{1}{\sigma\left(B_{k, j}^{i}\right)} \int_{B_{k, j}^{i}} \frac{f_{i}(y)}{\sigma(y)} \sigma(y) d y\right]^{q} \\
=c \sum_{k, j} \mu_{k, j}^{i}\left(g_{k, j}^{i}\right)^{q}
\end{gathered}
$$

where

$$
\mu_{k, j}^{i}=\left(w^{1 / r} g\right)\left(E_{k, j}^{i}\right)\left(\frac{1}{\left|B_{k, j}^{i}\right|} \int_{B_{k, j}^{i}} \sigma(y) d y\right)^{q}
$$

and

$$
g_{k, j}^{i}=\frac{1}{\sigma\left(B_{k, j}^{i}\right)} \int_{B_{k, j}^{i}} \frac{f_{i}(y)}{\sigma(y)} \sigma(y) d y .
$$

We view the sum $\sum_{k, j} \mu_{k, j}^{i}\left(g_{k, j}^{i}\right)^{q}$, as an integral on a measure space $\left(X, \mu^{i}\right)$ built over the set $X=\{k, j\}$, assigning to each $(k, j)$ the measure $\mu_{k, j}^{i}$. For $\lambda>0$, set

$$
\begin{gathered}
\Gamma^{i}(\lambda)=\left\{(k, j): g_{k, j}^{i}>\lambda\right\}, \\
\Omega^{i}(\lambda)=\bigcup_{(k, j) \in \Gamma^{i}(\lambda)} B_{k, j}^{i} .
\end{gathered}
$$


Then

$$
\sum_{k, j}\left(g_{k, j}^{i}\right)^{q} \mu_{k, j}^{i}=\int_{0}^{\infty} \lambda^{q} \mu^{i}\left(\Gamma^{i}(\lambda)\right) \frac{d \lambda}{\lambda} .
$$

Using (32) we can estimate $\mu^{i}\left(\Gamma^{i}(\lambda)\right)$ as follows:

$$
\begin{aligned}
\mu^{i}\left(\Gamma^{i}(\lambda)\right) & =\sum_{(k, j) \in \Gamma^{i}(\lambda)} \mu_{k, j}^{i} \\
& \leq \sum_{(k, j) \in \Gamma^{i}(\lambda)} \int_{E_{k, j}^{i}} M_{\mathcal{B}}\left(\sigma \chi_{B_{k, j}^{i}}\right)(y)^{q} w(y)^{1 / r} g(y) d y \\
& \leq \int_{\Omega^{i}(\lambda)} M_{\mathcal{B}}\left(\sigma \chi_{\Omega^{i}(\lambda)}\right)(y)^{q} w(y)^{1 / r} g(y) d y \\
& \leq c\left(\sigma^{1 / r} G\right)\left(\Omega^{i}(\lambda)\right) \leq c\left(\sigma^{1 / r} G\right)\left(\left\{y \in \mathbf{R}^{n}: M_{\mathcal{B}, \sigma}\left(\frac{f_{i}}{\sigma}\right)(y)>\lambda\right\}\right) .
\end{aligned}
$$

Hence,

$$
\begin{gathered}
I \leq c \sum_{i=0}^{\infty} \int_{0}^{\infty} \lambda^{q}\left(\sigma^{1 / r} G\right)\left(\left\{y \in \mathbf{R}^{n}: M_{\mathcal{B}, \sigma}\left(\frac{f_{i}}{\sigma}\right)(y)>\lambda\right\}\right) \frac{d \lambda}{\lambda} \\
=c \int_{\mathbf{R}^{n}} \sum_{i=0}^{\infty} M_{\mathcal{B}, \sigma}\left(\frac{f_{i}}{\sigma}\right)(y)^{q} \sigma(y)^{1 / r} G(y) d y \leq c\left\|\left(\sum_{i=0}^{\infty} M_{\mathcal{B}, \sigma}\left(\frac{f_{i}}{\sigma}\right)^{q}\right)^{1 / q}\right\|_{L^{p}(\sigma)}^{q}\|G\|_{L^{r^{\prime}}} \\
\leq c\left\|\left(\sum_{i=0}^{\infty}\left(\frac{f_{i}}{\sigma}\right)^{q}\right)^{1 / q}\right\|_{L^{p}(\sigma)}^{q}\|g\|_{L^{r^{\prime}}}=c\left\|\left(\sum_{i=0}^{\infty} f_{i}^{q}\right)^{1 / q}\right\|_{L^{p}(v)}^{q} .
\end{gathered}
$$

concluding the proof of the proposition.

We cannot apply immediately Proposition 2.1 to the basis of cubes $\mathcal{Q}$ since it is not true in general that $M_{\sigma}$ is bounded on $L_{\ell q}^{p}(\sigma)$; recall that for the scalar case we must assume that $\sigma$ is doubling. However, if we consider the basis of dyadic cubes $\mathcal{D}$ we can still prove that for all $1<p, q<\infty$,

$$
M_{\mathcal{D}, \mu}: L_{\ell^{q}}^{p}(\mu) \rightarrow L_{\ell^{q}}^{p}(\mu)
$$

for any positive Borel measure $\mu$ on $\mathbf{R}^{n}$. Indeed, this follows from

$$
\int_{\mathbf{R}^{n}} M_{\mathcal{D}, \mu} f(x)^{p} w(x) d \mu(x) \leq C \int_{\mathbf{R}^{n}}|f(x)|^{p} M_{\mathcal{D}, \mu} w(x) d \mu(x),
$$

which is a consequence of standard methods. Using this we have the following particular case of Proposition 2.1.

Proposition 2.2. Let $1<q<p<\infty, r=\frac{p}{q}$ and let $\sigma=v^{1-p^{\prime}}$.

Then the two weight vector-valued inequality

$$
\left\|\left(\sum_{i=0}^{\infty}\left(M_{\mathcal{D}} f_{i}\right)^{q}\right)^{1 / q}\right\|_{L^{p}(w)} \leq C\left\|\left(\sum_{i=0}^{\infty}\left|f_{i}\right|^{q}\right)^{1 / q}\right\|_{L^{p}(v)}
$$

holds if and only if there exists a constant $c$ such that for each $g \in L^{r^{\prime}}\left(\mathbf{R}^{n}\right)$ we can find $G \in L^{r^{\prime}}\left(\mathbf{R}^{n}\right)$ with $\|G\|_{L^{r^{\prime}}\left(\mathbf{R}^{n}\right)} \leq\|g\|_{L^{r^{\prime}}\left(\mathbf{R}^{n}\right)}$ such that 


$$
\int_{Q} M_{\mathcal{D}}\left(\sigma \chi_{Q}\right)(x)^{q} w(x)^{1 / r} g(x) d x \leq c \int_{Q} \sigma(x)^{1 / r} G(x) d x,
$$

for all dyadic cubes $Q$.

Proof. To prove the proposition we just need to see that condition (35) extends from dyadic cubes to arbitrary open sets which are a union of dyadic cubes. But to do this is simply a repetition of the argument given in [GCRdF, p. 430 and we shall omit it.

Finally we have all the ingredients to prove the main theorem of this section.

Theorem 2.3. Let $1<q<p<\infty, r=\frac{p}{q}$, and let $\sigma=v^{1-p^{\prime}}$. Then the two weight vector-valued inequality

$$
\int_{\mathbf{R}^{n}} \bar{M}_{q} f(x)^{p} w(x) d x \leq C \int_{\mathbf{R}^{n}}|f(x)|_{q}^{p} v(x) d x
$$

holds if and only there exists a constant $c$ such that for each $g \in L^{r^{\prime}}\left(\mathbf{R}^{n}\right)$ we can find $G \in L^{r^{\prime}}\left(\mathbf{R}^{n}\right)$ with $\|G\|_{L^{r^{\prime}}\left(\mathbf{R}^{n}\right)} \leq\|g\|_{L^{r^{\prime}}\left(\mathbf{R}^{n}\right)}$ such that

$$
\int_{Q} M\left(\sigma \chi_{Q}\right)(x)^{q} w(x)^{1 / r} g(x) d x \leq c \int_{Q} \sigma(x)^{1 / r} G(x) d x,
$$

for all cubes $Q$.

Proof. By Proposition 2.1 all we have to do is to prove that (37) implies (36).

Following GCRdF, p. 432 we shall use the following pointwise estimate:

For each integer $k$, each locally integrable function $f$, and each $x \in \mathbf{R}^{n}$, there is a constant $c$, depending only on the dimension $n$ such that the following pointwise inequality holds

$$
M^{2^{k}} f(x) \leq c \frac{1}{\mid Q_{2^{k+2}(0) \mid}} \int_{Q_{2^{k+2}(0)}}\left(\tau_{-t} \circ M_{\mathcal{D}} \circ \tau_{t}\right) f(x) d t,
$$

where $\tau_{t} g(x)=g(x-t), Q_{r}(0)$ is the cube centered at the origin with side length $r$, and $M^{\delta}, \delta>0$, is the operator defined as $M$ but with cubes having side length smaller than $\delta$.

Now by Minkowski's inequality and Fatou's Lemma we have

$$
\|M f\| \leq c \sup _{t>0}\left\|\left(\tau_{-t} \circ M_{\mathcal{D}} \circ \tau_{t}\right) f\right\|
$$

for any norm \|\| . Then the boundedness of $M$ is equivalent to the uniform boundedness of $\tau_{-t} \circ M_{\mathcal{D}} \circ \tau_{t}, t \in \mathbf{R}^{n}$, namely

$$
\left\|\left(\sum_{i=0}^{\infty}\left(\left(\tau_{-t} \circ M_{\mathcal{D}} \circ \tau_{t}\right) f_{i}\right)^{q}\right)^{1 / q}\right\|_{L^{p}(w)} \leq C\left\|\left(\sum_{i=0}^{\infty}\left|f_{i}\right|^{q}\right)^{1 / q}\right\|_{L^{p}(v)}
$$

with a constant $C$ independent of $t \in \mathbf{R}^{n}$. But this is equivalent to

$$
\left\|\left(\sum_{i=0}^{\infty}\left(M_{\mathcal{D}} f_{i}\right)^{q}\right)^{1 / q}\right\|_{L^{p}\left(\tau_{t} w\right)} \leq C\left\|\left(\sum_{i=0}^{\infty}\left|f_{i}\right|^{q}\right)^{1 / q}\right\|_{L^{p}\left(\tau_{t} v\right)} .
$$


Then we must show that $\left(\tau_{t} w, \tau_{t} v\right)$ satisfies condition (35) with a constant independent of $t$. Indeed, let $g \in L^{r^{\prime}}\left(\mathbf{R}^{n}\right)$, then

$$
\begin{gathered}
\int_{Q} M_{\mathcal{D}}\left(\left(\tau_{t} v\right)^{1-p^{\prime}} \chi_{Q}\right)(x)^{q}\left(\tau_{t} w\right)(x)^{1 / r} g(x) d x \\
=\int_{Q-t} \tau_{-t} \circ M_{\mathcal{D}} \circ \tau_{t}\left(\sigma \chi_{Q-t}\right)(x)^{q} w(x)^{1 / r} \tau_{-t} g(x) d x \\
\leq \int_{Q-t} M\left(\sigma \chi_{Q-t}\right)(x)^{q} w(x)^{1 / r} \tau_{-t} g(x) d x .
\end{gathered}
$$

By hypothesis there exists a function $G(x, t)=G_{t}(x)$ with

$$
\left\|G_{t}\right\|_{L^{r^{\prime}\left(\mathbf{R}^{n}\right)}} \leq\left\|\tau_{-t} g\right\|_{L^{r^{\prime}\left(\mathbf{R}^{n}\right)}}=\|g\|_{L^{r^{\prime}\left(\mathbf{R}^{n}\right)}}
$$

and such that the last integral is dominated by a constant, independent of $t$, times

$$
\int_{Q-t} \sigma(x)^{1 / r} G_{t}(x) d x=\int_{Q}\left(\tau_{t} v(x)\right)^{\left(1-p^{\prime}\right) / r} \tau_{t} G_{t}(x) d x .
$$

Observe that $\tilde{G}=\tau_{t} G_{t}$ verifies the hypothesis since $\|\tilde{G}\|_{L^{r^{\prime}}\left(\mathbf{R}^{n}\right)} \leq\|g\|_{L^{r^{\prime}\left(\mathbf{R}^{n}\right)}}$.

The class of pairs of weights defined by condition (37) is contained in Sawyer's condition (8) as the following argument shows. If $(w, v)$ satisfies (37), then there exists by the Hann-Banach theorem a function $g \in L^{r^{\prime}}\left(\mathbf{R}^{n}\right)$ with unit norm such that

$$
\begin{gathered}
\left(\int_{Q} M\left(\sigma \chi_{Q}\right)(x)^{p} w(x) d x\right)^{1 / r}=\left(\int_{Q}\left(M\left(\sigma \chi_{Q}\right)(x)^{q} w(x)^{1 / r}\right)^{r} d x\right)^{1 / r} \\
=\int_{Q} M\left(\sigma \chi_{Q}\right)(x)^{q} w(x)^{1 / r} g(x) d x .
\end{gathered}
$$

Then by (37) there exists $G \in L^{r^{\prime}}$ with $\|G\|_{L^{r^{\prime}}\left(\mathbf{R}^{n}\right)} \leq 1$ and such that this last expression is dominated by

$$
\begin{aligned}
& \leq c \int_{Q} \sigma(x)^{1 / r} G(x) d x \\
& \leq c\left(\int_{Q} \sigma(x) d x\right)^{1 / r}\left(\int_{\mathbf{R}^{n}} G(x)^{r^{\prime}} d x\right)^{1 / r^{\prime}} \\
& \leq c\left(\int_{Q} \sigma(x) d x\right)^{1 / r} .
\end{aligned}
$$

Moreover, condition (37) is strictly contained in $S_{p}$ as the counterexample $(w, M w)$ in (15) shows.

\section{The main tool: A generalization of the Hardy-Littlewood MAXIMAL FUNCTION AND ITS BOUNDEDNESS PROPERTIES}

The purpose of this section is to introduce a further variation of the HardyLittlewood function whose boundedness properties will be the key ingredient to obtain sharp weighted estimates in different situations as in the proof of Theorem 1.1 (see the next section) and also in the problem treated in Section 1.3 ,

Let $A:[0, \infty) \rightarrow[0, \infty)$ be an increasing function such that $A(0)=0$. Given a cube $Q$ on $\mathbf{R}^{n}$ we define the $A$-average of a function $f$ over $Q$ by means of the following Luxemburg type "norm"

$$
\|f\|_{A, Q}=\inf \left\{\lambda>0: \frac{1}{|Q|} \int_{Q} A\left(\frac{|f(y)|}{\lambda}\right) d y \leq 1\right\} .
$$


We define the corresponding maximal operator $M_{A}$ by

$$
M_{A} f(x)=\sup _{x \in Q}\|f\|_{A, Q},
$$

where the supremum is taken over all the cubes containing $x$. When $A(t)=t^{\epsilon}$ we get $M_{A}=M_{L^{\epsilon}}=M_{\epsilon}, \epsilon>0$, but more interesting examples are provided by Young functions like $A(t)=t \log ^{\epsilon}(1+t), \epsilon>0$ since we are close to the case $A(t)=t$ which corresponds to the Hardy-Littlewood maximal function.

If furthermore $A$ is a Young function, namely $A$ is continuous, convex and increasing, there exists a complementary Young function $\bar{A}$ that satisfies

$$
t \leq A^{-1}(t) \bar{A}^{-1}(t) \leq 2 t, \quad t>0 .
$$

A key fact is the following generalized Hölder's inequality:

$$
\frac{1}{|Q|} \int_{Q}|f(y) g(y)| d y \leq\|f\|_{A, Q}\|g\|_{\bar{A}, Q} .
$$

The relevant class of Young functions is the following.

Definition 3.1. Let $1<p<\infty$. We say that a doubling Young function $B$ satisfies the $B_{p}$ condition if there is a positive constant $c$ such that

$$
\int_{c}^{\infty} \frac{B(t)}{t^{p}} \frac{d t}{t}<\infty
$$

It is easy to see that this condition is equivalent to saying that

$$
\int_{c}^{\infty}\left(\frac{t^{p^{\prime}}}{\bar{B}(t)}\right)^{p-1} \frac{d t}{t}<\infty .
$$

Theorem 3.2. Let $1<p<\infty$. Then the following statements are equivalent.

i)

ii) there is a constant $c$ such that

$$
\int_{\mathbf{R}^{n}} M_{B} f(y)^{p} d y \leq c \int_{\mathbf{R}^{n}} f(y)^{p} d y
$$

for all nonnegative, locally integrable functions $f$;

iii) there is a constant $c$ such that

$$
\int_{\mathbf{R}^{n}} M_{B} f(y)^{p} w(y) d y \leq c \int_{\mathbf{R}^{n}} f(y)^{p} M w(y) d y
$$

for all nonnegative, locally integrable functions $f$, and $w$;

iv) there is a constant $c$ such that

$$
\int_{\mathbf{R}^{n}} M f(y)^{p} \frac{w(y)}{\left[M_{\bar{B}}\left(u^{1 / p}\right)(y)\right]^{p}} d y \leq c \int_{\mathbf{R}^{n}} f(y)^{p} \frac{M w(y)}{u(y)} d y,
$$

for all nonnegative functions $f, w$ and $u$;

v) let $1<s \leq \infty$, then

$$
M_{B}: L_{\ell^{s}}^{p}\left(\mathbf{R}^{n}\right) \rightarrow L_{\ell^{s}}^{p}\left(\mathbf{R}^{n}\right) .
$$

Proof. We give the proof of the theorem for completeness since it will appear in [P1] except for the last equivalence which is needed for the proof of Theorem 1.3.

We will show that i) $\Rightarrow$ ii) $\Rightarrow$ iii) $\Rightarrow$ iv) and then that i) $\Leftrightarrow \mathrm{v}$ )

For the proof that i) implies ii) we need the following lemma. 
Lemma 3.3. Suppose that $B$ is a Young function, and that $f$ is a nonnegative bounded function with compact support. For each $t>0$, let $\Omega_{t}=\left\{y \in \mathbf{R}^{n}\right.$ : $\left.M_{B} f(y)>t\right\}$. Then, if $\Omega_{t}$ is not empty, we have

$$
\Omega_{t} \subset \bigcup_{j} 3 Q_{j}
$$

where $Q_{j}$ is the family of nonoverlapping maximal dyadic cubes satisfying

$$
\frac{t}{4^{n}}<\|f\|_{B, Q_{j}} \leq \frac{t}{2^{n}}
$$

for each integer $j$.

Furthermore it follows that

$$
\left|\Omega_{t}\right| \leq C \int_{\left\{y \in \mathbf{R}^{n}: f(y)>t / 2\right\}} B\left(\frac{f(y)}{t}\right) d y
$$

and

$$
\left\{y \in \mathbf{R}^{n}: M_{B}^{d} f(y)>\frac{t}{4^{n}}\right\}=\bigcup_{j} Q_{j},
$$

where $M_{B}^{d}$ denotes the dyadic anlogue of $M_{B}^{d}$.

We defer the proof of the lemma for the moment, and assume i). To prove ii) we shall use the classical approach (cf. for instance [GCRdF] Ch. 2). Hence, (47) and the change of variable $t=\frac{f(y)}{s}$ yield

$$
\begin{gathered}
\int_{\mathbf{R}^{n}} M_{B} f(y)^{p} d y=p \int_{0}^{\infty} t^{p}\left|\left\{y \in \mathbf{R}^{n}: M_{B} f(y)>t\right\}\right| \frac{d t}{t} \\
\leq C \int_{0}^{\infty} t^{p} \int_{\left\{y \in \mathbf{R}^{n}: f(y)>t / 2\right\}} B\left(\frac{f(y)}{t}\right) d y \frac{d t}{t}=C \int_{\mathbf{R}^{n}} \int_{0}^{2 f(y)} t^{p} B\left(\frac{f(y)}{t}\right) \frac{d t}{t} d y \\
=C \int_{\mathbf{R}^{n}} f(y)^{p} d y \int_{1 / 2}^{\infty} \frac{B(t)}{t^{p}} \frac{d t}{t}=C \int_{\mathbf{R}^{n}} f(y)^{p} d y,
\end{gathered}
$$

since $B \in B_{p}$. This proves that i) implies ii).

For the proof that ii) implies iii) we discretize $M_{B}$ as follows. We fix a constant $a>2^{n}$, and for each integer $k$ we let $\Omega_{k}$, and $D_{k}$ be the sets

$$
\begin{aligned}
& \Omega_{k}=\left\{x \in \mathbf{R}^{n}: M_{B} f(x)>a^{k}\right\}, \\
& D_{k}=\left\{x \in \mathbf{R}^{n}: M_{B}^{d} f(x)>\frac{a^{k}}{4^{n}}\right\} .
\end{aligned}
$$

Here $M_{B}^{d}$ denotes the dyadic version of $M_{B}$. Hence, by Lemma 3.3 with $t=a^{k}$ there is a family of maximal nonoverlapping dyadic cubes $\left\{Q_{k, j}\right\}$ for which $\Omega_{k} \subset$ $\bigcup_{j} 3 Q_{k, j}, D_{k}=\bigcup_{j} Q_{k, j}$, and

$$
\frac{a^{k}}{4^{n}}<\|f\|_{B, Q_{k, j}} \leq \frac{a^{k}}{2^{n}}
$$

We shall need the following lemma.

Lemma 3.4. Suppose $a>2^{n}$. For all integers $k, j$ we let $E_{k, j}=Q_{k, j}-Q_{k, j} \cap D_{k+1}$. Then $\left\{E_{k, j}\right\}$ is a disjoint family of sets which satisfy

$$
\left|Q_{k, j} \cap D_{k+1}\right|<\frac{2^{n}}{a}\left|Q_{k, j}\right|,
$$


and

$$
\left|Q_{k, j}\right|<\frac{1}{1-\frac{2^{n}}{a}}\left|E_{k, j}\right| .
$$

We also postpone the proof of this lemma until the end of the proof of the theorem.

Now, using (49) and (51) we can estimate the left side of (43) as follows:

$$
\begin{gathered}
\int_{\mathbf{R}^{n}} M_{B} f(y)^{p} w(y) d y=\sum_{k} \int_{\Omega_{k}-\Omega_{k+1}} M_{B} f(y)^{p} w(y) d y \\
\leq a^{p} \sum_{k} a^{k p} w\left(\Omega_{k}\right) \leq C \sum_{k, j} a^{k p} w\left(3 Q_{k, j}\right) \\
\leq C \sum_{k, j}\|f\|_{B, Q_{k, j}}^{p} w\left(3 Q_{k, j}\right)=C \sum_{k, j}\|f\|_{B, Q_{k, j}}^{p} \frac{w\left(3 Q_{k, j}\right)}{\left|3 Q_{k, j}\right|}\left|Q_{k, j}\right| \\
\leq C \sum_{k, j}\left\|f\left(\frac{w\left(3 Q_{k, j}\right)}{\left|3 Q_{k, j}\right|}\right)^{1 / p}\right\|_{B, Q_{k, j}}^{p}\left|E_{k, j}\right| \\
\leq C \sum_{k, j} \int_{E_{k, j}} M_{B}\left(f(M w)^{1 / p}\right)(y)^{p} d y \leq C \int_{\mathbf{R}^{n}} M_{B}\left(f(M w)^{1 / p}\right)(y)^{p} d y \\
\leq C \int_{\mathbf{R}^{n}} f(y)^{p} M w(y) d y,
\end{gathered}
$$

since we are assuming ii). This proves iii).

Let us assume that iii) holds. Observe that (44) is equivalent to

$$
\int_{\mathbf{R}^{n}} M(f g)(y)^{p} \frac{w(y)}{\left[M_{\bar{B}}(g)(y)\right]^{p}} d y \leq c \int_{\mathbf{R}^{n}} f(y)^{p} M w(y) d y,
$$

for all nonnegative functions $f, g$, and $w$. Then iv) follows immediately from (43) after an application of the inequality

$$
M(f g)(y) \leq M_{B} f(y) M_{\bar{B}} g(y), \quad y \in \mathbf{R}^{n},
$$

which is a consequence of the generalized Hölder's inequality (41).

To prove that iv) implies i) we let $w=1$ in (44) obtaining

$$
\int_{\mathbf{R}^{n}} M f(y)^{p} \frac{1}{\left[M_{\bar{B}}\left(u^{1 / p}\right)(y)\right]^{p}} d y \leq c \int_{\mathbf{R}^{n}} f(y)^{p} \frac{1}{u(y)} d y,
$$

for all nonnegative functions $f$, and $u$. Testing this inequality with $f=u=\chi_{Q(0,1)}$, where $Q(x, r)$ denotes the cube centered at $x \in \mathbf{R}^{n}$ and with sidelength equal to $r$, we have

$$
\int_{\mathbf{R}^{n}} M f(y)^{p} \frac{1}{\left[M_{\bar{B}}(f)(y)\right]^{p}} d y \leq C .
$$

On the other hand, we have for large $x$ that

$$
M_{\bar{B}}(f)(x) \approx \frac{1}{\bar{B}^{-1}\left(\frac{1}{|x|^{n}}\right)} .
$$


Therefore we get

$$
\begin{gathered}
\int_{\mathbf{R}^{n}} M f(y)^{p} \frac{1}{\left[M_{\bar{B}}(f)(y)\right]^{p}} d y \geq C \int_{|y|>c} \frac{1}{|y|^{n p}} \frac{1}{\bar{B}^{-1}\left(\frac{1}{\left.|y|^{n}\right)^{p}} d y\right.} \\
=C \int_{c}^{\infty} \frac{1}{r^{n p}} \frac{1}{\bar{B}^{-1}\left(\frac{1}{r^{n}}\right)^{p}} r^{n} \frac{d r}{r} \approx \int_{c}^{\infty} \frac{B(t)}{t^{p}} \frac{d t}{t} .
\end{gathered}
$$

This estimate combined with (53) shows that iv) $\Rightarrow \mathrm{i}$ ).

To conclude the proof of the theorem, apart from the proofs of Lemmas 3.3 and 3.4, we need to show that i) $\Leftrightarrow \mathrm{v}$ ). That i) is necessary is trivial since v) implies the scalar case, namely ii). To show that i) is sufficient observe that the case $p<s$ follows by interpolation from the cases $s=p, s=\infty$. Now the case $p>s$ follows from the weighted inequality iii) by standard arguments.

Proof of Lemma 3.3. The proof is a simple adaptation of arguments in [GCRdF], Ch. 2. Since $f$ is bounded with compact support, say $\operatorname{supp} f \subset K$,

$$
\|f\|_{B, Q} \leq\|f\|_{L^{\infty}}\left\|\chi_{K}\right\|_{B, Q}=\|f\|_{L^{\infty}} \frac{1}{B^{-1}\left(\frac{|Q|}{|Q \cap K|}\right)},
$$

and it follows that

$$
\|f\|_{B, Q} \rightarrow 0
$$

as $Q \uparrow \mathbf{R}^{n}$. Hence, if there are any dyadic cubes $Q$ with $\|f\|_{B, Q}>t$, they are contained in cubes of this type which are maximal with respect to inclusion. We let $C_{t}=\left\{P_{j}\right\}$ be the family of the dyadic maximal nonoverlapping cubes satisfying

$$
t<\|f\|_{B, P_{j}} .
$$

Let $P_{j}^{\prime}$ be the only dyadic cube containing $P_{j}$ with sidelength twice that of $P_{j}$. Then

$$
t<\|f\|_{B, P_{j}} \leq 2^{n}\|f\|_{B, P_{j}^{\prime}} .
$$

The last inequality can easily be deduced from the definition of the Luxemburg norm using the fact that $t \rightarrow \frac{B(t)}{t}$ is nondecreasing. Hence by the maximality of the cubes $\left\{P_{j}\right\}$ we get

$$
t<\|f\|_{B, P_{j}} \leq 2^{n} t
$$

Observe that from this discussion it is clear that

$$
\left\{y \in \mathbf{R}^{n}: M_{B}^{d} f(y)>t\right\}=\bigcup_{j} P_{j} .
$$

Let $x \in \Omega_{t}$. By definition, there is a cube $R$ containing $x$ such that

$$
t<\|f\|_{B, R} .
$$

Let $k$ be the unique integer such that $2^{-(k+1) n}<|R| \leq 2^{-k n}$. There is some dyadic cube with side length $2^{-k}$, and at most $2^{n}$ of them, $\left\{J_{i}: i=1, \ldots, n\right\}$, meet the interior of $R$. It is easy to see that for one of these cubes, say $J_{1}$,

$$
\frac{t}{2^{n}}<\left\|\chi_{J_{1}} f\right\|_{B, R} .
$$


This can be seen as follows. If for each $i=1, \ldots, 2^{n}$ we had

$$
\left\|\chi_{J_{i}} f\right\|_{B, R} \leq \frac{t}{2^{n}}
$$

we would get since $R \subset \bigcup_{i=1}^{2^{n}} J_{i}$ that

$$
\begin{gathered}
\|f\|_{B, R}=\| \chi_{\cup_{\cup_{i=1}^{2 n} J_{i}} f \|_{B, R}} \\
\leq \sum_{i=1}^{2^{n}}\left\|\chi_{J_{i}} f\right\|_{B, R} \leq 2^{n} \frac{t}{2^{n}}=t,
\end{gathered}
$$

contradicting (56). Since $|R| \leq\left|J_{1}\right|<2^{n}|R|$ one can also show

$$
\frac{t}{4^{n}}<\|f\|_{B, J_{1}} .
$$

By letting $C_{t /(4)^{n}}=\left\{Q_{j}\right\}$, we have by (54) that

$$
\frac{t}{4^{n}}<\|f\|_{B, Q_{j}} \leq \frac{t}{2^{n}},
$$

for each $j$, yielding (46). Equation (48) also follows since $\left\{y \in \mathbf{R}^{n}: M_{B}^{d} f(y)>\right.$ $\left.\frac{t}{4^{n}}\right\}=\bigcup_{j} Q_{j}$. Also, we see from (58) that $J_{1} \subset Q_{k}$, for some $k$, and then $R \subset$ $3 J_{1} \subset 3 Q_{k}$. This gives

$$
\Omega_{t} \subset \bigcup_{j} 3 Q_{j},
$$

which is (45). Now, by the left side of the inequality (59), and the definition of $\|f\|_{B, Q}$ we get

$$
\begin{gathered}
\left|\Omega_{t}\right| \leq C \sum_{j}\left|Q_{j}\right| \\
\leq C \sum_{j} \int_{Q_{j}} B\left(\frac{4^{n} f(y)}{t}\right) d y \leq C \int_{\mathbf{R}^{n}} B\left(\frac{f(y)}{t}\right) d y .
\end{gathered}
$$

To obtain (47) we just use the standard idea of writing $f$ as $f=f_{1}+f_{2}$, where $f_{1}(x)=f(x)$ if $f(x)>\frac{t}{2}$, and $f_{1}(x)=0$ otherwise. Then $M_{B} f(x) \leq$ $M_{B} f_{1}(x)+M_{B} f_{2}(x) \leq M_{B} f_{1}(x)+\frac{t}{2}$. Finally, since (60) holds for each $f \geq 0, t>0$ we have

$$
\begin{aligned}
\left|\Omega_{t}\right| \leq \mid\{y & \left.\in \mathbf{R}^{n}: M_{B} f_{1}(y)>\frac{t}{2}\right\} \mid \leq C \int_{\mathbf{R}^{n}} B\left(\frac{f_{1}(y)}{t}\right) d y \\
& =C \int_{\left\{y \in \mathbf{R}^{n}: f(y)>t / 2\right\}} B\left(\frac{f(y)}{t}\right) d y,
\end{aligned}
$$

concluding the proof of Lemma 3.3 .

We now conclude the proof of the theorem by proving Lemma 3.4 .

Proof of Lemma 3.4. The family $E_{k, j}$ is clearly disjoint. We note that (49) and the definition of the Luxemburg norm implies that

$$
1<\frac{1}{\left|Q_{k, j}\right|} \int_{Q_{k, j}} B\left(\frac{4^{n}}{a^{k}} f(y)\right) d y,
$$


and

$$
\frac{1}{\left|Q_{k, j}\right|} \int_{Q_{k, j}} B\left(\frac{2^{n}}{a^{k}} f(y)\right) d y \leq 1
$$

Hence by standard properties of the dyadic cubes we can estimate what portion of $Q_{k, j}$ is covered by $D_{k+1}$ as in [GCRdF], p. 398

$$
\begin{gathered}
\frac{\left|Q_{k, j} \cap D_{k+1}\right|}{\left|Q_{k, j}\right|}=\sum_{i} \frac{\left|Q_{k, j} \cap Q_{k+1, i}\right|}{\left|Q_{k, j}\right|}=\sum_{i: Q_{k+1, i} \subset Q_{k, j}} \frac{\left|Q_{k+1, i}\right|}{\left|Q_{k, j}\right|} \\
\quad<\sum_{i: Q_{k+1, i} \subset Q_{k, j}} \frac{1}{\left|Q_{k, j}\right|} \int_{Q_{k+1, i}} B\left(\frac{4^{n}}{a^{k+1}} f(y)\right) d y \\
\leq \frac{2^{n}}{a} \frac{1}{\left|Q_{k, j}\right|} \int_{Q_{k, j} \cap \cup_{i} Q_{k+1, i}} B\left(\frac{2^{n}}{a^{k}} f(y)\right) d y \leq \frac{2^{n}}{a} .
\end{gathered}
$$

Here we have used that $B\left(\frac{2^{n}}{a} t\right) \leq \frac{2^{n}}{a} B(t), t>0$, since $\frac{2^{n}}{a}<1$, and because $t \rightarrow \frac{B(t)}{t}$ is increasing. This gives (50). Finally

$$
\frac{\left|E_{k, j}\right|}{\left|Q_{k, j}\right|}>1-\frac{2^{n}}{a}>0
$$

completing the proof of the lemma and hence that of Theorem 3.2 .

\section{Proof of the main theOrem*}

In this section we give the proof of Theorem 1.1. We start with the proof of part a), the positive part. We apply Theorem 2.3 by verifying condition (37). The weight $w$ is fixed and $v$ will be chosen in a moment. Recall that $r=\frac{p}{q}$ and that $\sigma=v^{1-p^{\prime}}$. We need to show that there exists a constant $c$ such that for arbitrary $g \in L^{r^{\prime}}\left(\mathbf{R}^{n}\right)$ there is $G \in L^{r^{\prime}}\left(\mathbf{R}^{n}\right)$ with $\|G\|_{L^{r^{\prime}}\left(\mathbf{R}^{n}\right)} \leq\|g\|_{L^{r^{\prime}}\left(\mathbf{R}^{n}\right)}$ and such that for all cubes $Q$

$$
\int_{Q} M\left(\sigma \chi_{Q}\right)(x)^{q} w(x)^{1 / r} g(x) d x \leq c \int_{Q} \sigma(x)^{\frac{1}{r}} G(x) d x .
$$

We use the Fefferman-Stein inequality (10) together with the generalized Hölder's inequality (41) to estimate the left hand side of (61) by a multiple of

$$
\begin{gathered}
\int_{Q} \sigma(x)^{q} M\left(w^{1 / r} g\right)(x) d x \leq \int_{Q} \sigma(x)^{q} M_{B}\left(w^{1 / r}\right)(x) M_{\bar{B}}(g)(x) d x \\
=\int_{Q} \sigma(x)^{q}\left[\left(M_{B}\left(w^{1 / r}\right)(x)\right)^{r}\right]^{1 / r} M_{\bar{B}}(g)(x) d x .
\end{gathered}
$$

If we let $v=\left(M_{B}\left(w^{1 / r}\right)\right)^{r}$ we have that

$$
\int_{Q} M\left(\sigma \chi_{Q}\right)(x)^{q} w(x)^{1 / r} g(x) d x \leq C \int_{Q} \sigma(x)^{1 / r} M_{\bar{B}}(g)(x) d x .
$$

To conclude the proof all we have to do know is to choose $B$ such that $\bar{B} \in B_{r^{\prime}}$, namely that

$$
\int_{c}^{\infty}\left(\frac{t^{r}}{B(t)}\right)^{r^{\prime}-1} \frac{d t}{t}<\infty
$$

\footnotetext{
* Note added in proof: We found a different more direct proof in [CP].
} 
since by the characterization in Theorem $3.2 M_{\bar{B}}: L^{r^{\prime}}\left(\mathbf{R}^{n}\right) \rightarrow L^{r^{\prime}}\left(\mathbf{R}^{n}\right)$. If we let $\left\|M_{\bar{B}}\right\|$ be the norm of this operation we can take $G=M_{\bar{B}}(g) /\left\|M_{\bar{B}}\right\|$ such that $\|G\|_{L^{r^{\prime}}\left(\mathbf{R}^{n}\right)} \leq\|g\|_{L^{r^{\prime}}\left(\mathbf{R}^{n}\right)}$ and we have

$$
\int_{Q} M\left(\sigma \chi_{Q}\right)(x)^{q} w(x)^{1 / r} g(x) d x \leq C\left\|M_{\bar{B}}\right\| \int_{Q} \sigma(x)^{1 / r} G(x) d x .
$$

Finally we are left with showing that we can pick $B$ such that $v=\left(M_{B}\left(w^{1 / r}\right)\right)^{r} \leq$ $M^{[r]+1} w$. Indeed, let $B(t) \approx t^{r}(\log (1+t))^{[r]}$, then $B\left(t^{1 / r}\right) \approx t\left(\log (1+t)^{[r]}\right.$ and $\left(M_{B}\left(w^{1 / r}\right)\right)^{r}=M_{L(\log L)^{[r]}}(w)$. Now, we make the following observation.

Let $k=1,2,3, \cdots$, then there exists a constant $C=C_{n}$ such that for all bounded functions $f$ with support contained in $Q$

$$
\|f\|_{L(\log L)^{k}, Q} \leq \frac{C}{|Q|} \int_{Q} M^{k} f(y) d y .
$$

Indeed, by homogeneity we can assume that the right hand side is one. Then by the definition of the Luxemburg norm it is enough to prove

$$
\frac{1}{|Q|} \int_{Q} f(y)\left(1+\log ^{+}(f(y))\right)^{k} d y \leq C,
$$

which is a consequence of iterating the following well known inequality of E.M. Stein:

$$
\int_{Q} f(y)\left(1+\log ^{+}(f(y))\right)^{k} d y \leq C \int_{Q} M f(y)\left(1+\log ^{+}(M f(y))\right)^{k-1} d y,
$$

with $k=1,2,3, \cdots$.

Therefore we finally have that $v=M_{L(\log L)[r]}(w) \leq M^{[r]+1} w$ concluding the proof of the theorem.

This concludes the proof of the first part, for the counterexample we take $n=1$ and we let $N$ be a large positive integer and $r=\frac{p}{q}>1$. Set $w=\chi_{(0,1)}$ and define

$$
f_{i}(x)=(\log x)^{-1 / q} \chi_{\left(e^{i}, e^{i+1}\right)}(x)
$$

for each $i=1, \cdots, N-1$, and $f_{i}=0$ for $i \geq N$. Then,

$$
\begin{gathered}
\left\|\left(\sum_{i=1}^{\infty}\left|f_{i}\right|^{q}\right)^{1 / q}\right\|_{L^{p}\left(M^{[r]} w\right)}^{p}=\int_{\mathbf{R}}\left(\sum_{i=1}^{N-1}(\log x)^{-1} \chi_{\left(e^{i}, e^{i+1}\right)}(x)\right)^{r} M^{[r]} w(x) d x \\
\approx \int_{e}^{e^{N}}(\log x)^{-r}(\log x)^{[r]-1} \frac{d x}{x} .
\end{gathered}
$$

When $r$ is an integer this is comparable to $\log N$, and when $r$ is not an integer the integral is a constant independent of $N$ since $[r]-r+1>0$. In any case, it is less than a constant times $\log N$. On the other hand,

$$
\begin{gathered}
\left\|\left(\sum_{i=1}^{\infty}\left(M f_{i}\right)^{q}\right)^{1 / q}\right\|_{L^{p}(w)}^{p}=\int_{0}^{1}\left(\sum_{i=1}^{N-1}\left(M f_{i}(x)\right)^{q}\right)^{r} d x \\
\geq \int_{0}^{1}\left(\sum_{i=1}^{N-1} \frac{1}{i}\right)^{r} d x \approx(\log N)^{r}
\end{gathered}
$$


since for $0<x<1$ and $i=1, \cdots, N-1$,

$$
M f_{i}(x) \geq \frac{1}{e^{i+1}} \int_{0}^{e^{i+1}}(\log y)^{-1 / q} \chi_{\left(e^{i}, e^{i+1}\right)}(y) d y \approx \frac{1}{i^{1 / q}} .
$$

To conclude, observe that $(\log N)^{r} \leq C \log N$ does not make sense for large $N$ with $C$ independent of $N$.

We conclude the section by giving the following simple argument showing that $M w \in A_{1}$ assuming that $w \in A_{\infty}$ which was used to prove inequality (16). Indeed since $w$ satisfies for some $r>1$ the reverse Hölder inequality

$$
\left(\frac{1}{|Q|} \int_{Q} w^{r}\right)^{1 / r} \leq \frac{C}{|Q|} \int_{Q} w
$$

with $C$ independent of the cube $Q$. Now for fixed $Q$ and $x \in Q$ we have

$$
\begin{gathered}
\frac{1}{|Q|} \int_{Q} M w \leq \frac{1}{|Q|} \int_{Q} M\left(w \chi_{2 Q}\right)+\frac{1}{|Q|} \int_{Q} M\left(w \chi_{\mathbf{R}^{n} \backslash 2 Q}\right) \\
\leq\left(\frac{1}{|Q|} \int_{Q} M\left(w \chi_{2} Q\right)^{r}\right)^{1 / r}+C \inf _{Q} M(w) \leq C\left(\frac{1}{|2 Q|} \int_{2 Q} w^{r}\right)^{1 / r}+M(w)(x) \\
\leq \frac{C}{|2 Q|} \int_{2 Q} w+M(w)(x) \leq C M(w)(x) .
\end{gathered}
$$

Here we have used that $M\left(\chi_{\mathbf{R}^{n} \backslash 2 Q} w\right)(y) \approx M\left(\chi_{\mathbf{R}^{n} \backslash 2 Q} w\right)(z)$ for each $y, z \in Q$, GCRdF, p. 159 and the $L^{r}$ boundedness of $M$. This means that $M w \in A_{1}$.

\section{Proof of the Sharp Sufficient Conditions}

We want to point out that we do not know how to prove this theorem directly from the characterization given in Theorem 2.3 . We are going to modify and combine the proof of this theorem with the results in Theorem 3.2 which in fact contains the key estimate.

Proof of Theorem 1.3 .

$$
\left\|\left(\sum_{i=0}^{\infty}\left(w M f_{i}\right)^{q}\right)^{1 / q}\right\|_{L^{p}\left(\mathbf{R}^{n}\right)}^{q}=\sum_{i=0}^{\infty} \int_{\mathbf{R}^{n}} M f_{i}(y)^{q} w(y)^{q} g(y) d y
$$

for some $g \in L^{r^{\prime}}\left(\mathbf{R}^{n}\right)$ with unit norm.

Let $i$ be fixed. For each integer $k$, and for any arbitrary constant $a>2^{n}$ we let $\Omega_{k}^{i}$, and $D_{k}^{i}$ be the sets

$$
\begin{gathered}
\Omega_{k}^{i}=\left\{x \in \mathbf{R}^{n}: a^{k}<M f_{i}(x)\right\}, \\
D_{k}^{i}=\left\{x \in \mathbf{R}^{n}: M^{d} f_{i}(x)>\frac{a^{k}}{4^{n}}\right\} .
\end{gathered}
$$

By the classical Calderón-Zygmund decomposition (cf. [GCRdF, p. 137) there exists a family of maximal nonoverlapping dyadic cubes $\left\{Q_{k, j}^{i}\right\}$ for which $\Omega_{k}^{i} \subset$ $\bigcup_{j} 3 Q_{k, j}^{i}, D_{k}^{i}=\bigcup_{j} Q_{k, j}^{i}$, and

$$
\frac{a^{k}}{4^{n}}<\frac{1}{\left|Q_{k, j}^{i}\right|} \int_{Q_{k, j}^{i}} f_{i}(y) d y \leq \frac{a^{k}}{2^{n}}
$$


We can now estimate the integral in (63) as follows:

$$
\begin{gathered}
\int_{\mathbf{R}^{n}} M f_{i}(y)^{q} w(y)^{q} g(y) d y=\sum_{k} \int_{\Omega_{k}^{i}-\Omega_{k+1}^{i}} M f_{i}(y)^{q} w(y)^{q} g(y) d y \\
\leq a^{q} \sum_{k} a^{k q}\left(w^{q} g\right)\left(\Omega_{k}^{i}\right) \leq C \sum_{k, j} a^{k q}\left(w^{q} g\right)\left(3 Q_{k, j}^{i}\right) \\
\leq C \sum_{k, j}\left(\frac{1}{\left|Q_{k, j}^{i}\right|} \int_{Q_{k, j}^{i}} f_{i}(y) d y\right)^{q}\left(w^{q} g\right)\left(3 Q_{k, j}^{i}\right) \\
=C \sum_{k, j}\left(\frac{1}{\left|Q_{k, j}^{i}\right|} \int_{Q_{k, j}^{i}} f_{i}(y) v(y) v(y)^{-1} d y\right)^{q}\left(w^{q} g\right)\left(3 Q_{k, j}^{i}\right) \\
\leq C \sum_{k, j}\left(\frac{1}{\left|3 Q_{k, j}^{i}\right|} \int_{3 Q_{k, j}^{i}} f_{i}(y) v(y) v(y)^{-1} d y\right)^{q} \frac{1}{\left|3 Q_{k, j}^{i}\right|} \int_{3 Q_{k, j}^{i}} w(y)^{q} g(y) d y\left|Q_{k, j}^{i}\right| .
\end{gathered}
$$

For each integer $k, j$ we set $E_{k, j}^{i}=Q_{k, j}^{i}-Q_{k, j}^{i} \cap D_{k+1}^{i}$. Then $\left\{E_{k, j}^{i}\right\}$ is a disjoint family of sets, and by Lemma 3.3 with $B(t)=t$, there is a positive constant $\beta$ such that for each $k, j\left|Q_{k, j}^{i}\right|<\beta\left|E_{k, j}^{i}\right|$. This together with the generalized Hölder's inequality (41) allows us to dominate the last sum by a multiple of

$$
\begin{gathered}
\sum_{k, j}\left\|v f_{i}\right\|_{\bar{B}, 3 Q_{k, j}^{i}}^{q}\left\|v^{-1}\right\|_{B, 3 Q_{k, j}^{i}}^{q}\left\|w^{q}\right\|_{A, 3 Q_{k, j}^{i}}\|g\|_{\bar{A}, 3 Q_{k, j}^{i}}\left|E_{k, j}^{i}\right| \\
\leq K^{q} \sum_{k, j} \int_{E_{k, j}^{i}} M_{\bar{B}}\left(v f_{i}\right)(y)^{q} M_{\bar{A}}(g)(y) d y \leq C \int_{\mathbf{R}^{n}} M_{\bar{B}}\left(v f_{i}\right)(y)^{q} M_{\bar{A}}(g)(y) d y
\end{gathered}
$$

since the sets $\left\{E_{k, j}^{i}\right\}$ are pairwise disjoint when $i$ is fixed. Hence, by Hölder's inequality with exponents $r$ and $r^{\prime}$ we can estimate 63) by

$$
\begin{gathered}
\left\|\left(\sum_{i=0}^{\infty}\left(w M f_{i}\right)^{q}\right)^{1 / q}\right\|_{L^{p}\left(\mathbf{R}^{n}\right)}^{q} \leq C\left\|\left(\sum_{i=0}^{\infty}\left(M_{\bar{B}}\left(f_{i} v\right)\right)^{q}\right)^{1 / q}\right\|_{L^{p}\left(\mathbf{R}^{n}\right)}^{q}\left\|M_{\bar{A}}(g)\right\|_{L^{r^{\prime}\left(\mathbf{R}^{n}\right)}} \\
\leq C\left\|\left(\sum_{i=0}^{\infty}\left(v f_{i}\right)^{q}\right)^{1 / q}\right\|_{L^{p}\left(\mathbf{R}^{n}\right)}^{q}\|g\|_{L^{r^{\prime}\left(\mathbf{R}^{n}\right)}}=\left\|\left(\sum_{i=0}^{\infty}\left(v f_{i}\right)^{q}\right)^{1 / q}\right\|_{L^{p}\left(\mathbf{R}^{n}\right)}^{q}
\end{gathered}
$$

since $M_{\bar{B}}: L_{\ell^{q}}^{p}\left(\mathbf{R}^{n}\right) \rightarrow L_{\ell^{q}}^{p}\left(\mathbf{R}^{n}\right)$ and $M_{\bar{A}}: L^{r^{\prime}}\left(\mathbf{R}^{n}\right) \rightarrow L^{r^{\prime}}\left(\mathbf{R}^{n}\right)$ by Theorem 3.2

\section{Endpoint EStimates And the Besicovitch LEMma}

Proof of Theorem 1.4. It is enough to consider $f \geq 0$ in the sense that $f_{i} \geq 0$ for all $i$. Let $\Omega=\left\{x \in \mathbf{R}^{n}: M^{d}\left(|f|_{q}\right)(x)>\lambda\right\}=\bigcup Q$, where the dyadic cubes $Q$ are maximal nonoverlapping satisfying

$$
\lambda<\frac{1}{|Q|} \int_{Q}|f(x)|_{q} d x \leq 2^{n} \lambda,
$$

and $|f(x)|_{q} \leq \lambda$, a.e. $x \in \mathbf{R}^{n} \backslash \Omega$. Write $f=f \cdot \chi_{\mathbf{R}^{n}-\Omega}+f \cdot \chi_{\Omega}=g+b$. Since $\bar{M}_{q} f \leq \bar{M}_{q} g+\bar{M}_{q} b$, it is sufficient to estimate the distribution set of $\bar{M}_{q} g$ and $\bar{M}_{q} b$ separately. 
Observing that $|g(x)|_{q} \leq \lambda$ we get by (10)

$$
\begin{gathered}
w\left(\left\{x \in \mathbf{R}^{n}: \bar{M}_{q} g(x)>\lambda / 2\right\}\right) \leq \frac{C}{\lambda^{q}} \int_{\mathbf{R}^{n}} \bar{M}_{q} g(x)^{q} w(x) d x \\
\leq \frac{C}{\lambda^{q}} \sum_{i} \int_{\mathbf{R}^{n}}\left(M g_{i}\right)(x)^{q} w(x) d x \leq \frac{C}{\lambda^{q}} \sum_{i} \int_{\mathbf{R}^{n}} g_{i}(x)^{q} M w(x) d x \\
=\frac{C}{\lambda^{q}} \int_{\mathbf{R}^{n}}|g(x)|_{q}^{q} M w(x) d x \leq \frac{C}{\lambda} \int_{\mathbf{R}^{n}}|f(x)|_{q} M w(x) d x .
\end{gathered}
$$

For $b$ we split the distibution set of $\bar{M}_{q} b$ as follows. Let $\tilde{\Omega}=\bigcup \tilde{Q}, \tilde{Q}=3 Q$. Then

$$
w\left(\left\{x \in \mathbf{R}^{n}: \bar{M}_{q} b(x)>\lambda / 2\right\}\right) \leq w\left(\left\{x \in \mathbf{R}^{n} \backslash \tilde{\Omega}: \bar{M}_{q} b(x)>\lambda / 2\right\}\right)+w(\tilde{\Omega}) .
$$

The second term is estimated by the left hand side of (66):

$$
\begin{aligned}
w(\tilde{\Omega}) & \leq \frac{C}{\lambda} \sum_{Q} w(\tilde{Q}) \frac{1}{|Q|} \int_{Q}|f(x)|_{q} d x \\
\leq & \frac{C}{\lambda} \sum_{Q} \int_{Q}|f(x)|_{q} M w(x) d x \\
& \leq \frac{C}{\lambda} \int_{\mathbf{R}^{n}}|f(x)|_{q} M w(x) d x .
\end{aligned}
$$

For the first term we use the argument in [FS], p. 110 which shows that $M b_{i}(x) \leq$ $M\left(\bar{b}_{i}\right)(x), x \in \mathbf{R}^{n} \backslash \tilde{\Omega}$ where $\bar{b}_{i}$ is the function

$$
\begin{gathered}
\bar{b}_{i}(x)= \begin{cases}0, & x \in \mathbf{R}^{n} \backslash \Omega, \\
\frac{1}{|Q|} \int_{Q} f_{i}(y) d y, & x \in Q,\end{cases} \\
w\left(\left\{x \in \mathbf{R}^{n} \backslash \tilde{\Omega}: \bar{M}_{q} b(x)>\lambda / 2\right\} \leq \frac{1}{\lambda^{q}} \sum_{i} \int_{\mathbf{R}^{n} \backslash \tilde{\Omega}}\left(M b_{i}(x)\right)^{q} w(x) d x\right. \\
\leq \frac{1}{\lambda^{q}} \sum_{i} \int_{\mathbf{R}^{n} \backslash \tilde{\Omega}}\left(M\left(\bar{b}_{i}\right)(x)\right)^{q} w(x) d x \leq \frac{C}{\lambda^{q}} \sum_{i} \int_{\mathbf{R}^{n}} \bar{b}_{i}(x)^{q} M\left(w \chi_{\mathbf{R}^{n} \backslash \tilde{\Omega}}\right)(x) d x \\
=\frac{C}{\lambda^{q}} \sum_{i} \sum_{Q}\left(\frac{1}{|Q|} \int_{Q} f_{i}(y) d y\right)^{q} \int_{Q} M\left(w \chi_{\mathbf{R}^{n} \backslash \tilde{Q}}\right)(x) d x \\
\leq \frac{C}{\lambda^{q}} \sum_{Q}\left[\sum_{i}\left(\frac{1}{|Q|} \int_{Q} f_{i}(y) d y\right)^{q}\right] \int_{Q}^{\frac{1}{q} q} M\left(w \chi_{\mathbf{R}^{n} \backslash \tilde{Q}}\right)(x) d x \\
\leq \frac{C}{\lambda^{q}} \sum_{Q}\left[\frac{1}{|Q|} \int_{Q}|f(y)|_{q} d y\right]^{q} \inf _{Q} M(w)|Q| \leq \frac{C}{\lambda} \sum_{Q} \frac{1}{|Q|} \int_{Q}|f(y)|_{q} d y \inf _{Q} M(w)|Q| \\
\leq \frac{C}{\lambda} \sum_{Q} \int_{Q}|f(x)|_{q} M w(x) d x \leq \frac{C}{\lambda} \int_{\mathbf{R}^{n}}|f(x)|_{q} M w(x) d x .
\end{gathered}
$$


As we mentioned in the introduction there exists a close connection between the weighted scalar inequality

$$
w\left(\left\{x \in \mathbf{R}^{n}: M f(x)>\lambda\right\}\right) \leq \frac{C}{\lambda} \int_{\mathbf{R}^{n}}|f(x)| M w(x) d x
$$

and the the Besicovitch covering lemma $[\mathrm{dG}$. Indeed, the first observation is that 67 is equivalent to

$$
w\left(\left\{x \in \mathbf{R}^{n}: M\left(f \frac{w}{M w}\right)(x)>\lambda\right\}\right) \leq \frac{C}{\lambda} \int_{\mathbf{R}^{n}}|f(x)| w(x) d x .
$$

The second is that we trivially have the pointwise inequality

$$
M\left(f \frac{w}{M w}\right)(x) \leq c_{n} M_{w}^{c} f(x)
$$

where $M_{w}^{c}$ is the weighted centered maximal function

$$
M_{w}^{c} f(x)=\sup _{r>0} \frac{1}{w\left(B_{r}(x)\right)} \int_{B_{r}(x)}|f(y)| w(y) d y .
$$

Therefore (67) follows from

$$
w\left(\left\{x \in \mathbf{R}^{n}: M_{w}^{c} f(x)>\lambda\right\}\right) \leq \frac{C}{\lambda} \int_{\mathbf{R}^{n}}|f(x)| w(x) d x
$$

which is a consequence of the Besicovitch covering lemma.

We can repeat this argument with $M$ replaced by the vector-valued maximal operator $\bar{M}_{q}$ except for the fact that there is no vector-valued analogue of the Besicovitch lemma, namely

$$
w\left(\left\{x \in \mathbf{R}^{n}:\left(\sum_{i=1}^{\infty}\left(M_{w}^{c} f_{i}(x)\right)^{q}\right)^{1 / q}>\lambda\right\}\right) \leq \frac{C}{\lambda} \int_{\mathbf{R}^{n}}|f(x)|_{q} w(x) d x .
$$

Combining this estimate together with (68) would yield a different proof of Theorem 1.4.

\section{REFERENCES}

[AJ] K. F. Andersen and R. T. John, Weighted inequalities for vector-valued maximal functions and singular integrals, Studia Math. 69 (1980), 19-31. MR 82b:42015

[BCP] A. Benedek, A. P. Calderón, and R. Panzone, Convolution operators on Banach space valued functions, Proc. Nat. Acad. Aci. USA 48, (1962), 356-365. MR 24:A3479

[CP] D. Cruz-Uribe, SFO and C. Pércz, Two weight extrapolation via the maximal operator, preprint.

[CWW] S. Y. A. Chang, J. M. Wilson, and T. H. Wolff, Some weighted norm inequalities concerning the Schrödinger operators, Comment. Math. Helvetici 60 (1985), 217-286. MR 87d:42027

[CW] S. Chanillo and R. Wheeden, Some weighted norm inequalities for the area integral, Indiana Univ. Math. J. 36 (1987). MR 88e:42036

[FS] C. Fefferman and E. M. Stein, Some maximal inequalities, Amer. J. Math. 93 (1971), 107-115. MR 44:2026

[GCRdF] J. Garcia-Cuerva and J. L. Rubio de Francia, Weighted norm inequalities and related topics, North-Holland Math. Studies 116, North-Holland, Amsterdam, (1985). MR 87d:42023

[dG] M. de Guzmán, Differentiation of Integrals in $\mathbf{R}^{n}$, Lect. Notes in Math. 481, SpingerVerlag, (1975). MR 56:15866

[K] V. Kokilashvili, Maximal inequalities and multipliers in weighted Lizorkin-Triebel spaces, Soviet Math. Dokl. 19 (2), 272-276. 
[M] B. Muckenhoupt, Weighted norm inequalities for the Hardy maximal function, Trans. Amer. Math. Soc. 165 (1972), 207-226. MR 45:2461

[N] C. J. Neugebauer, Inserting $A_{p}$-weights, Proc. Amer. Math. Soc. 87 (1983), 644-648. MR 84d:42026

[P1] C. Pérez, On sufficient conditions for the boundedness of the Hardy-Littlewood maximal operator between weighted $L^{p}$-spaces with different weights. Proc. London Math. Soc. (3) 71 (1995), 135-157. MR 96k:42023

[P2] C. Pérez, Weighted norm inequalities for singular integral operators, J. London Math. Soc. 49 (1994), 296-308. MR 94m:42037

[R] Y. Rakotondratsimba, A characterization of a two weight norm vector-valued inequality for maximal operators, Georgian Math. J. 5 (1998), 583-600.

[RRT] J. L. Rubio de Francia, F. J. Ruiz, and J. L. Torrea, Calderón-Zygmund theory for operator-valued kernels, Adv. in Math. 62, (1986), 7-48. MR 88f:42035

[S] E. T. Sawyer, A characterization of a two-weight norm inequality for maximal operators, Studia Math. 75 (1982), 1-11. MR 84i:42032

[Wil1] J. M. Wilson, A sharp inequality for the square function, Duke Math. Journal. 55 (1987), 879-887. MR 89a:42029

[Wil2] J. M. Wilson, Weighted norm inequalities for the continuous square function, Trans. Amer. Math. Soc. 314 (1989), 661-692. MR 91e:42026, MR 91e:42025

[Wil3] J. M. Wilson, $L^{p}$ weighted norm inequalities for the square function, $0<p<2$, Illinois Journal of Mathematics. 33 (1989), 361-666. MR 90g:42037

[Wil4] J. M. Wilson, Chanillo-Wheeden inequalities for $0<p \leq 1$, J. London Math. Soc. 41 (1990), 283-294. MR 91j:42018

Departmento de Matemáticas, Universidad Autónoma de Madrid, 28049 Madrid, Spain

E-mail address: carlos.perez@uam.es 